\title{
Cyberchondria, Uncertainty, and Psychological Distress among Omanis during COVID-19: An Online Cross-Sectional Survey
}

Khloud Al Dameery

Sultan Qaboos University

Mohammed Quteshat

Sultan Qaboos University

Ibtisam Al Harthy

Sultan Qaboos University

Atika Khalaf ( $\square$ atika.khalaf@hkr.se)

Kristianstad University \& Sultan Qaboos University https://orcid.org/0000-0002-9958-0222

\section{Research Article}

Keywords: Cyberchondria, Uncertainty, Psychological Distress, COVID 19

Posted Date: September 29th, 2020

DOI: https://doi.org/10.21203/rs.3.rs-84556/v1

License: (c) (i) This work is licensed under a Creative Commons Attribution 4.0 International License. Read Full License 


\section{Abstract}

Background: Frequent surfing on the internet for self-diagnosis and treatment in association with quarantine during the COVID-19 pandemic leaves people with uncertainty and psychological distress. The aim was to explore the relationship between cyberchondria, psychological distress, and uncertainty in Oman.

Methods: A descriptive correlational study was designed. Data was collected through an online survey from 393 Omani participants using the short-form version of the Cyberchondria Severity Scale, the Kessler Psychological Distress Scale, and the Intolerance of Uncertainty Scale. Descriptive and correlational analyses were conducted.

Results: The majority of the participants were aged between $18-26$ and $45-54$ years (32.8\% and $30.8 \%$ respectively). Almost three-quarters of the participants were female $(n=291,74 \%)$ and half were single $(n=218,55.5 \%)$. No significant associations were found between cyberchondria and the sociodemographic factors or the COVID-19 status. However, significant positive fair correlation was found between cyberchondriac experiences and psychological distress $\left(r_{s}=0.373\right)$, and cyberchondria and uncertainty $\left(r_{s}=0.442\right)$.

Conclusions: To the best of our knowledge, this study is the first of its kind investigating the relationship between cyberchondria, physiological distress, and uncertainty in the Omani population. Policy makers and stakeholders are encouraged to facilitate the reporting and observation of accurate and up-to-date information distributed to the population to avoid misleading information, and thus reduce the distress and uncertainty related to cyberchondriac behaviors.

\section{Background}

With increased global health challenges and health illiteracy, individuals face new problems related to their health and the availability of the seemingly endless amount of internet-based information, with a possible increase in uncertainty, distress, and anxiety in relation to information-seeking behaviours. More people are using the internet as a source of knowledge about wellbeing. The estimated monthly retrieval of electronic health information for adults in the United States is $62 \%$, making it the fifth most popular online activity [1]. People seek the health-related websites for many reasons, such as learning about a particular disease and its treatment; comparing their prescribed treatment with others; exploring other higher-quality medication than that prescribed for them; shopping for non-prescription medicines and herbal remedies; and hunting for discounted products [2]. Nevertheless, people benefit from these interactive online services by joining therapy groups and chatting with those who are having similar symptoms to express feelings and share ideas [3]. While it is considered as a great technological benefit as well as a time- and money-saver, surfing the internet for health-related information can have unfortunate consequences when it becomes the only source of diagnosis and treatment for individuals, increasing their anxiety about their health. The intensive or repetitive online searching for health 
information of particular real or imagined symptoms of illness is a new concept, known as cyberchondria, with potential negative psychological consequences for individuals [2, 4-6]. That is, when the individual looks on the internet for information related to their health concerns, this tends to increase their anxiety and distress [7]. A signature aspect of this disorder is compulsivity in the use of internet searching, which affects essential daily activities. It is understood that people with cyberchondria have limited health knowledge, leading them to seek information related to their condition and/or confirming their sickness. As a result of extensive internet searching, they are overwhelmed by the quantity of information retrieved, increasing their psychological distress [4]. Another explanation is that there is a group of people who are subject to internet illness-related attentional bias in which they continue searching until they selectively find themselves sick, which eventually increases their psychological distress. Cyberchondria can also be explained in the light of uncertainty. The in-depth searching and retrieval of a variety of health-related resources leave the individual with uncertainty and therefore more psychological distress [4]. Uncertainty pervades and motivates all practices relevant to healthcare. At its most basic, it is complexity of one type or another that promotes medical study, induces patients to pursue treatment, and promotes medical practice $[4,8]$. Meanwhile, social perception and confusion has been heightened by the increase in mainstream media reporting of medical controversies, from drug safety to brain tumour screening. Uncertainty affects every part of patient treatment, including the history of collecting and understanding clinical symptoms, choosing a method of investigation, working out the factors of the differential diagnoses and determining the result of a condition $[9,10]$.

Psychological distress is an important measure of mental health. It is defined as a state of emotional suffering that has distinctive features, such as symptoms of depression and anxiety [11]. It is associated with several health problems like cardiovascular, endocrine, and gastrointestinal and arthritic diseases [12].

There is little literature exploring cyberchondria among people in the Middle East. However, with convenient access to the Internet, the availability of health-related information and the psychological distress associated with quarantine and the mysterious nature of COVID-19, the number of people with cyberchondria is likely to increase. Our aim is to examine the relationship between cyberchondria, physiological distress and uncertainty in Oman.

\section{Methods}

\section{Data collection}

A descriptive correlational study design was used to examine the relationship between cyberchondria, uncertainty, and psychological distress in Oman. A sample of 393 participants was recruited using a convenience sampling technique (non-probability sampling). With the aim of minimising the costs and time for the data collection, the researchers recruited the participants through social media. The sample consisted of those who met the eligibility requirements: (a) living in Oman, (b) willing to participate in the study, and (c) signing an informed consent for participating in the study. 
Once ethical approval was obtained from all the required committees, the information sheet and survey questions were posted on social media platforms. The online survey, which was open throughout April 2020, took each participant about 20-25 minutes to complete.

\section{Ethical considerations}

Prior to data collection, ethical approval was obtained from the College of Nursing Scientific Research and Ethics Committee, Sultan Qaboos University (Ref.nr.: CON/NF/2020/15). The survey page was introduced with a short description of the nature, objectives, methodology, and potential benefits of the survey, ensuring the respondents' voluntary and confidential participation. By answering the survey, the participants gave their consent. No personal data was collected, so the participants' confidentiality was not an issue. All electronic data was saved on a password-protected computer, and only the members of the research team were able to identify the responses of individual subjects. The participants with COVID19 have not been reported in any other submission by this author team and to our knowledge not by anyone else.

\section{Study Instruments}

The researchers used a self-reporting questionnaire to measure the extents of the research phenomena, consisting of (1) demographic data; (2) the short-form version of the Cyberchondria Severity Scale (CSS12); (3) the Kessler Psychological Distress Scale (K10); and (4) the Intolerance of Uncertainty Scale, short form (IUS-12).

\section{The short-form version of the Cyberchondria Severity Scale}

The CSS-12 is a brief, reliable, and valid tool to measure worry/anxiety attributable to excessive online health search [13]. The scale consists of 12 items that are rated on a 5-point Likert-scale ranging from 1 ("never") to 5 ("always"). An example item is "If I notice an unexplained bodily sensation, I will search for it on the internet." The CSS-12 scale demonstrated reliable internal consistency as stated by the authors, with higher scores indicating increased levels of awareness [13]. Permission to use the survey was obtained from the owner of the instrument prior to the data collection.

\section{Kessler Psychological Distress Scale}

The K10 scale is composed of ten questions concerning emotional states, measuring psychological distress. Each item has a response scale of five levels, from $1=$ "never" to $5=$ "all of the time". The total score is the sum of the 10 questions, and a high score indicates a serious level of psychological distress. The scale demonstrates the strength of test-retest correlation coefficients, temporal stability, and internal consistency [14]. The survey is available in the public domain with permission to use if for non-profit purposes.

\section{Intolerance of Uncertainty Scale, short form}


This scale was developed by Carleton, Norton, and Asmundson (2007) to assess the responses of individuals to uncertainty and ambiguous situations. It consists of 12 items rated on a five-point Likert scale from 1 (not at all characteristic of me) to 5 (entirely characteristic of me). Possible scores range between 12 and 60, with higher scores reflecting greater uncertainty. The scale demonstrated good validity and reliability (Cronbach's alpha is 0.85 ) [15]. Permission to use the survey was obtained in advance from the owner.

\section{Statistical analysis plan}

The data was downloaded from the Google forms in Excel format, then transferred to the Statistical Package for the Social Sciences (SPSS) program version 23 for the analysis. All variables, including age were on categorical level except for the total scores of the questionnaires (CSS-12, K10, and IUS-12). Means, standard deviations, percentages, and frequencies were used to describe the participants' demographic information. Independent t-test and Spearman correlation were used to draw conclusions. The statistical significance was set at $p<0.05$.

The Independent t-test was performed to explore whether there is a significant difference between the mean score of the cyberchondria experience as the dependent variable and selected sample characteristics as independent variables. The Spearman correlation coefficient was used to test for the bivariate relationship between the dependent variable cyberchondria experience and the dependent variables of experienced psychological distress, uncertainty, and hours spent surfing the internet.

\section{Results}

Three hundred and ninety-three participants completed the online survey. The majority were aged between $18-26$ and $45-54$ years old (32.8\% and $30.8 \%$ respectively). Almost three-quarters of the participants were female $(n=291,74 \%)$, half were single $(n=218,55.5 \%)$, three-fifths have a university degree $(n=236,60.1 \%)$, and two-fifths receive less than 160 RO monthly $(n=162,41.2 \%)$. Furthermore, a large majority of the participants have no history of chronic illness $(n=341,86.8 \%)$, mental illness $(n=$ $375,95.4 \%)$, or a family history of mental illness ( $n=318,80.9 \%)$. A very small number $(n=11,2.8 \%)$ of the participants confirmed themselves as a positive COVID-19 case; one in ten ( $n=37,9.4 \%)$ had been quarantined, and three-fifths $(n=236,60.1 \%)$ thought that they were at high risk of COVID-19 infection. For more detail, see Table 1.

Table 1. Sample characteristics $(n=393)$. 


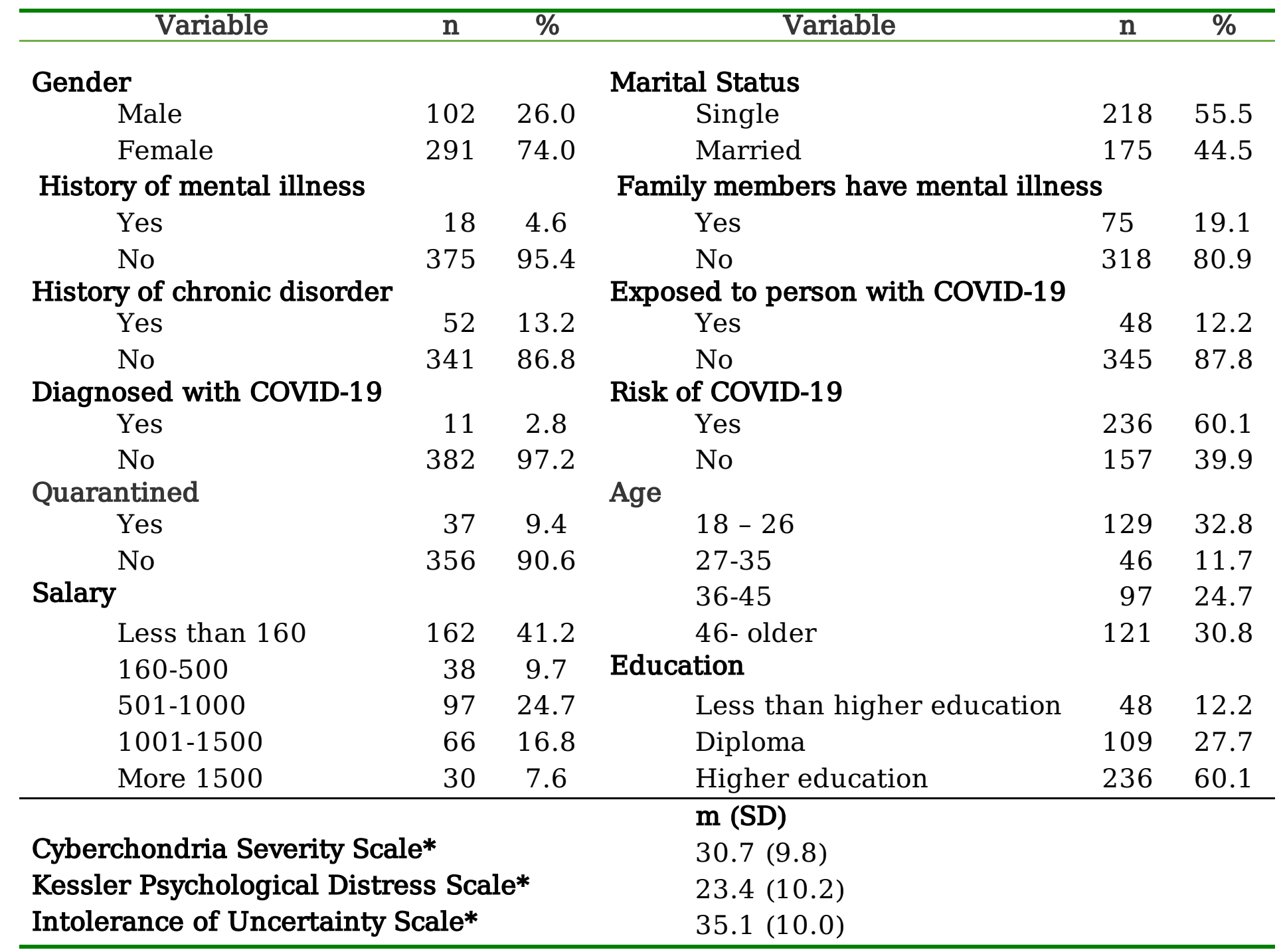

* min-max range for Cyberchondria Severity Scale is 12-60, Kessler Psychological Distress Scale is 10-50, and Intolerance of Uncertainty Scale is 12-60. Higher scores indicate greater severity, distress, and uncertainty.

In the group comparison phase, there was no statistically significant difference between cyberchondriac experience and gender $(p=0.595)$; marital status $(p=0.600)$; history of mental illness $(p=0.166)$; history of chronic illness ( $p=0.585)$; being diagnosed with COVID-19 ( $p=0.093)$; or being at risk from COVID-19 $(p=0.276)($ Table 2$)$.

Table 2: Relation between cyberchondriac experiences and socio-demographic factors. 


\begin{tabular}{|c|c|c|c|}
\hline Variable & Category & $\begin{array}{l}\text { Depression } \\
\mathrm{m}(S D)\end{array}$ & $p$ \\
\hline Gender & $\begin{array}{l}\text { Male } \\
\text { Female }\end{array}$ & $\begin{array}{l}30.2(9.4) \\
30.8(10)\end{array}$ & 0.595 \\
\hline Marital status & $\begin{array}{l}\text { single } \\
\text { married }\end{array}$ & $\begin{array}{l}30.4(10.4) \\
30.9(9)\end{array}$ & 0.600 \\
\hline History of mental illness & $\begin{array}{l}\text { No } \\
\text { Yes }\end{array}$ & $\begin{array}{l}30.5(9.7) \\
33.7(11.1)\end{array}$ & 0.166 \\
\hline Family member with mental illness & $\begin{array}{l}\text { No } \\
\text { Yes }\end{array}$ & $\begin{array}{l}30.3(9.7) \\
32(9.9)\end{array}$ & 0.537 \\
\hline History of chronic illness & $\begin{array}{l}\text { No } \\
\text { Yes }\end{array}$ & $\begin{array}{l}30.7(9.7) \\
30.2(10.3)\end{array}$ & 0.585 \\
\hline Exposed to person with COVID-19 & $\begin{array}{l}\text { No } \\
\text { Yes }\end{array}$ & $\begin{array}{l}30.9(9.5) \\
29(11.3)\end{array}$ & 0.216 \\
\hline Diagnosed with COVID-19 & $\begin{array}{l}\text { No } \\
\text { Yes }\end{array}$ & $\begin{array}{l}30.5(9.7) \\
35.5(11.8)\end{array}$ & 0.093 \\
\hline Being at risk of COVID-19 & $\begin{array}{l}\text { No } \\
\text { Yes }\end{array}$ & $\begin{array}{l}31.3(9.7) \\
30.2(9.8)\end{array}$ & 0.276 \\
\hline I have been quarantined for 14 days & $\begin{array}{l}\text { No } \\
\text { Yes }\end{array}$ & $\begin{array}{l}30.6(9.5) \\
31(12.2)\end{array}$ & 0.783 \\
\hline
\end{tabular}

A Pearson correlation analysis indicated that there was a significant positive fair correlation between cyberchondriac experiences, psychological distress $\left(r_{s}=0.373\right)$, and uncertainty $\left(r_{s}=0.442\right)$ (Table 3$)$.

Table 3. Correlations between cyberchondria experiences, psychological distress, uncertainty, and hours spent using the internet.

\begin{tabular}{lcccc}
\hline & 1 & 2 & 3 & 4 \\
\hline 1. Cyberchondriac experiences & 1 & $.373^{* *}$ & $.442^{* *}$ & .029 \\
2. Psychological distress & $.373^{* *}$ & 1 & $.586^{* *}$ & .018 \\
3. Uncertainty & $.442^{* *}$ & $.586^{* *}$ & 1 & .015 \\
Hours spent surfing the internet & .029 & .018 & .015 & 1 \\
\hline
\end{tabular}

\section{Discussion}


The aim of this study was to investigate the relationship between cyberchondria, psychological distress and uncertainty among the adult population of Oman. Our results show no significant difference between the cyberchondria and the socio-demographic factors. However, there is a significant fair correlation between cyberchondria and psychological distress as well as uncertainty.

The relationship between cyberchondria and psychological distress has been studied previously, and our findings are similar to those from other populations in the region. The most recently published study from Bahrain [16] showed that information-seeking behaviours through the internet caused moderate to severe anxiety problems in $18 \%$ of the participants during the COVID-19 pandemic. They further showed that the frequency of exposure to COVID-19 information was significantly associated with general anxiety disorders [16]. Although our sample answered questions about general health-seeking behaviours, our results show that cyberchondria is related to psychological distress. Regardless of the fact that cyberchondria is compulsive, reassurance often characterises online information-seeking behaviours [17].

Cyberchondria and uncertainty were found to have a fair association in our sample. No previous studies have investigated this relationship in the Middle Eastern context, although international studies have shown that inhibitory uncertainty has significant associations with cyberchondria dimensions [5]. Evidence for a unique relationship between the intolerance of uncertainty inhibitory factors and mistrust of medical professionals, which was interpreted as a cyberchondria-relevant construct [18]. Thus, the relationship between cyberchondria and uncertainty is an important aspect to consider when encountering anxious or worried patients in clinical settings. Healthcare providers need to adjust their approach to the needs of patients to gain their trust and thus their adherence and compliance to the medical and self-care guidance.

There were no significant associations between cyberchondria and socio-demographic factors in our sample. These findings are in line with other studies showing, for example, no association between gender and cyberchondria [6] or between age and cyberchondria [19]. However, similar accurate studies have identified positive associations between cyberchondria, health anxiety, and COVID-19 anxiety [20], anxiety and health information-seeking behaviours [16], and cyberchondria and gender [19]. The results of the current study suggest the need for wider investigation of socio-demographic factors, such as level of education and health literacy, on the information-seeking behaviours of different populations.

It is worth mentioning that the results of the current study are somewhat limited by the nature of the cross-sectional design, the convenience sampling technique, and self-reporting, all of which may affect the generalisability of the results to other populations. However, the variety of the study sample in relation to age and gender distribution may be considered as representative of the local Omani population.

\section{Conclusions}

The current study's findings shed some light on the status of cyberchondria in the general Omani population, with implications for further studies, practical healthcare provision, and policy making. In future research, the current study should be repeated, with the sample size increased, to confirm our 
findings. Further, for the healthcare and public health sectors, emphasis should be on the possible distress and uncertainty that cyberchondria may lead to, especially in times of global pandemics, hence affect the relationship between the healthcare provider and the client. Last but not least, policy makers and stakeholders need to develop reliable sources of health information at the national level, creating a social media platform that provides accurate and up-to-date information about the current situation, and taking measures to combat any source spreading of misinformation through the traditional or social media.

\section{Abbreviations}

Cyberchondria Severity Scale-12

Kessler Psychological Distress Scale-10

Intolerance of Uncertainty Scale, short form
CSS-12

K10

IUS-12

\section{Declarations}

\section{Ethics approval and consent to participate}

Prior to data collection, ethical approval was obtained from the College of Nursing Scientific Research and Ethics Committee, Sultan Qaboos University (Ref.nr.: CON/NF/2020/15). The survey page was introduced with a short description of the nature, objectives, methodology, and potential benefits of the survey, ensuring the respondents' voluntary and confidential participation. The informed consent was obtained through information on the first survey page in which it was clearly stated that the participants will give their written consent to participate by clicking the link to the survey, entering the survey page, answering the survey, and submitting it.

\section{Consent for publication}

Not applicable.

\section{Availability of data and materials}

The datasets generated and/or analyzed during the current study are available from the corresponding author on reasonable request.

\section{Competing interests}

The authors declare that they have no competing interests.

\section{Funding}

This research did not receive any funding. 
Authors' contributions

All authors contributed to the study conception and design. Material preparation and data collection were performed by $[K D],[M Q]$, and $[I H]$ and $[A K]$. The analysis was conducted by $[K D]$ and $[A K]$. Likewise, the first draft of the manuscript was written by $[K D]$ and $[A K]$. All authors commented on previous versions of the manuscript as well as read and approved the final manuscript.

\section{Acknowledgements}

Not applicable.

\section{References}

1. Taylor H, Poll H: Cyberchondriacs" on the Rise. The Harris Poll 2010, 95.

2. Durak Batıgün A, Gör N, Kömürcü B, Ertürk iş: Cyberchondria Scale (CS): Development, validity and reliability study. 2018.

3. Starcevic V, Berle D: Cyberchondria: towards a better understanding of excessive health-related Internet use. Expert Review of Neurotherapeutics 2013, 13(2):205-213.

4. Makarla S, Gopichandran V, Tondare D: Prevalence and correlates of cyberchondria among professionals working in the information technology sector in Chennai, India: A cross-sectional study. Journal of postgraduate medicine 2019, 65(2):87.

5. Fergus TA: Cyberchondria and intolerance of uncertainty: examining when individuals experience health anxiety in response to Internet searches for medical information. Cyberpsychology, Behavior, and Social Networking 2013, 16(10):735-739.

6. Fergus TA, Spada MM: Cyberchondria: Examining relations with problematic Internet use and metacognitive beliefs. Clinical psychology \& psychotherapy 2017, 24(6):1322-1330.

7. Te Poel F, Baumgartner SE, Hartmann T, Tanis M: The curious case of cyberchondria: A longitudinal study on the reciprocal relationship between health anxiety and online health information seeking. Journal of anxiety disorders 2016, 43:32-40.

8. Han PK, Klein WM, Arora NK: Varieties of uncertainty in health care: a conceptual taxonomy. Medical Decision Making 2011, 31(6):828-838.

9. Tyagi A, Garudkar S, Gagare A, Thopte A: Medical Uncertainty: Are we better off in era of evidence based medicine? International Journal of Medical Research \& Health Sciences 2015, 4(1):208-213.

10. Tyagi A, Haan C: Uncertainty analysis using corrected first-order approximation method. Water Resources Research 2001, 37(6):1847-1858.

11. Payton AR: Mental health, mental illness, and psychological distress: same continuum or distinct phenomena? Journal of health and Social Behavior 2009, 50(2):213-227.

12. Winchester RJ, Williams JS, Wolfman TE, Egede LE: Depressive symptoms, serious psychological distress, diabetes distress and cardiovascular risk factor control in patients with type 2 diabetes. 
Journal of Diabetes and its Complications 2016, 30(2):312-317.

13. McElroy E, Kearney M, Touhey J, Evans J, Cooke Y, Shevlin M: The CSS-12: Development and validation of a short-form version of the cyberchondria severity scale. Cyberpsychology, Behavior, and Social Networking 2019, 22(5):330-335.

14. Andrews G, Slade T: Interpreting scores on the Kessler psychological distress scale (K10). Australian and New Zealand journal of public health 2001, 25(6):494-497.

15. Carleton RN, Norton MPJ, Asmundson GJ: Fearing the unknown: A short version of the Intolerance of Uncertainty Scale. Journal of anxiety disorders 2007, 21(1):105-117.

16. Ebrahim A, Saif Z, Buheji M, AlBasri N, Al-Husaini F, Jahrami H: COVID-19 Information-Seeking Behavior and Anxiety Symptoms among Parents. OSP Journal of Health Care and Medicine 2020, 1(1):1-9.

17. Vismara M, Caricasole V, Starcevic V, Cinosi E, Dell'Osso B, Martinotti G, Fineberg NA: Is cyberchondria a new transdiagnostic digital compulsive syndrome? A systematic review of the evidence. Comprehensive Psychiatry 2020:152167.

18. Norr AM, Albanese BJ, Oglesby ME, Allan NP, Schmidt NB: Anxiety sensitivity and intolerance of uncertainty as potential risk factors for cyberchondria. Journal of Affective Disorders 2015, 174:6469.

19. Laato $S$, Islam AN, Islam MN, Whelan E: What drives unverified information sharing and cyberchondria during the COVID-19 pandemic? European Journal of Information Systems 2020:1-18.

20. Jungmann SM, Witthöft M: Health anxiety, cyberchondria, and coping in the current COVID-19 pandemic: Which factors are related to coronavirus anxiety? Journal of Anxiety Disorders 2020:102239. 